\title{
Experimental Characterization of a Graded-Index Ring- Core Fiber Supporting 7 LP Mode Groups
}

\author{
Feng Feng' ${ }^{1}$, George S. D. Gordon' ${ }^{1}$, X.Q. Jin², D.C. O'Brien ${ }^{2}$, F.P. Payne', Y. Jung ${ }^{3}$, Q. Kang ${ }^{3}$, \\ J.K. Sahu ${ }^{3}$, S.U. Alam ${ }^{3}$, D.J. Richardson ${ }^{3}$, Timothy D. Wilkinson ${ }^{1}$ \\ 1-Electrical Division, Engineering Department, University of Cambridge, 9, JJ Thomson Avenue, Cambridge, CB3 OFA, UK \\ 2-University of Oxford, Parks Road, Oxford, OX1 3PJ, UK \\ 3-Optoelectronics Research Centre, University of Southampton, Southampton, SO17 1BJ, UK \\ Corresponding email: ff263@cam.ac.uk.
}

\begin{abstract}
We design and characterize a graded-index-ring-core fiber supporting 7 LP modegroups (13 spatial modes) for mode multiplexed transmission with low MIMO processing complexity. Spatial and temporal modal properties are analyzed using an SLM-based mode multiplexer/demultiplexer.

OCIS codes: (060.2270) Fiber characterization; (060.4230) Multiplexing; (070.6120) Spatial light modulators.
\end{abstract}

\section{Introduction}

Mode-division multiplexing(MDM) has attracted much research interest as a means to further increase the transmission capacity of a single fiber by exploiting space as an additional degree of freedom[1,2]. Coupling between spatial modes is a major issue limiting the performance of MDM transmission, but the resultant scrambled signal can be recovered through multiple-input multiple-output (MIMO) signal processing [1]. Here the computational complexity and resultant energy consumption of the MIMO processing is especially important and specially designed fibers have been employed to minimize differential group delay (DGD). Strong coupling between spatial modes with small DGD can actually be beneficial, because mode-dependent losses or gains are averaged. Another option for fiber design is to use few mode fibers with large effective index difference ( $\Delta n_{\text {eff }}$ ) between modes, which can greatly suppress coupling between modes [3], in principle removing the need for MIMO processing entirely over a certain transmission distance.

In this paper, we present the first design and experimental characterization of a graded-index ring-core fiber (GIRCF) having a relatively large effective refractive index difference between mode groups based on a theoretical study of RCF design in [4]. This fiber is designed to support 7 LP mode groups such that the propagation constant difference $\Delta \beta$ between adjacent mode groups significantly increases with increasing azimuthal index number of the modes for the same radial mode index number. This design results in the DGD between low-order modes being significantly smaller than the DGD between high-order modes so that low-order mode channels require only low complexity MIMO processing and high-order mode channels do not require any MIMO processing within a certain transmission distance, although MIMO can be applied within a mode group if one wishes to use the degenerate modes to further increase capacity. Following this, the mode transfer matrix of two sample lengths, $5 \mathrm{~m}$ and $150 \mathrm{~m}$ respectively, is experimentally measured in two orthogonal polarizations using liquid crystal on silicon spatial light modulator (LCoS SLM) based multiplexer and de-multiplexer system. A time-domain analysis of the fiber's performance is presented and it is shown that isolations of $20 \mathrm{~dB}$ between adjacent high-order (>3) mode groups can be achieved.

\section{Graded-Index Ring-Core Fiber Design}
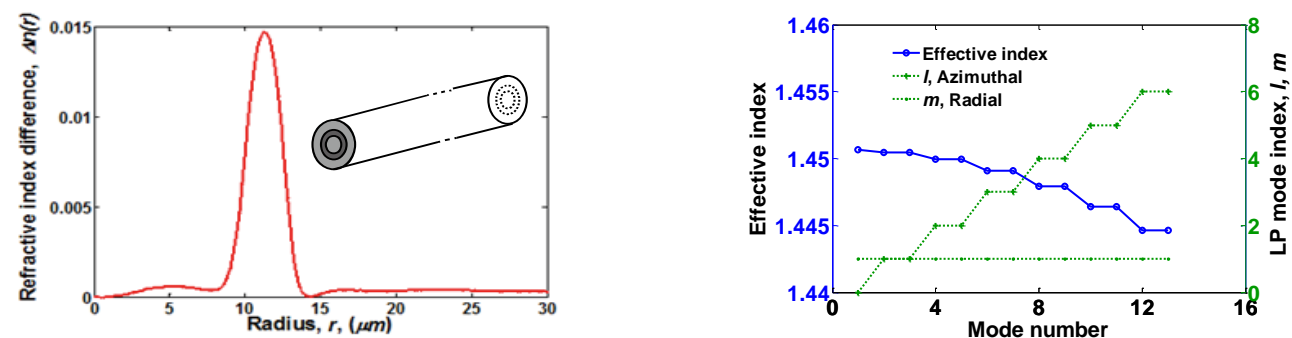

Figure 1 Measured refractive index profile of fabricated GI-RCF Figure 2 Effective index of all supported modes of the fiber The number of modes supported by a ring core fiber can be adjusted by changing the radius and thickness of the ring layer. The number of radial modes supported by the GI-RCF is designed to be one in order to use guided modes with large $\Delta n_{e f f}$ efficiently, and the number of azimuthal modes is set to be seven. This is achieved by a ring layer with 
average thickness $\sim 5 \mathrm{um}$ at an averaged radius $\sim 11 \mathrm{um}$. The measured refractive index profile of the fabricated GIRCF is illustrated in Figure 1. The refractive index of cladding layers is 1.444 at a wavelength of $1.55 u$ m. By numerically solving the scalar wave equation, the electric field, propagation constant and relative mode delay can be obtained. Figure 2 shows the theoretical effective index of all guided modes (7 LP mode groups) of the GI-RCF design and the theoretical mode filed distributions are shown in Figure 3.

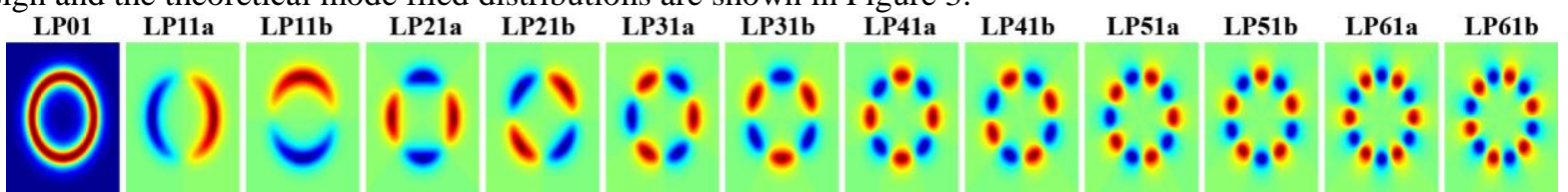

Figure 3 Theoretical mode field distributions of all guided modes of GI-RCF including degenerate modes.

\section{Experimental characterization of the GI-RCF}

The experimental system used to characterize the GI-RCF consists of LCoS SLM based multiplexer, demultiplexer and polarization diversity optics as illustrated in Figure 4 (a). Phase masks (holograms) that generate the precise modal field of each mode in the replay field for both multiplexer and demultiplexer are calculated under the constraint of $<1 \%$ error with maximum possible efficiency using a simulated annealing algorithm. An example of computer generated phase mask, for LP51a, is shown in Figure 4(b). A $1550 \mathrm{~nm}$ laser is modulated by MachZehnder modulator (MZM) driven by a vector network analyzer (VNA) which is capable of measuring from 40 $\mathrm{MHz}$ up to $20 \mathrm{GHz}$. The polarization of the signal is adjusted with a polarization controller to have equal power horizontal and vertical components before the multiplexer. After the demultiplexer, the received signal is split into two: one component of which goes to a photodiode connected to the VNA and the other goes to a power meter so that the frequency/time response and mode transfer matrix can be measured simultaneously.

(a)

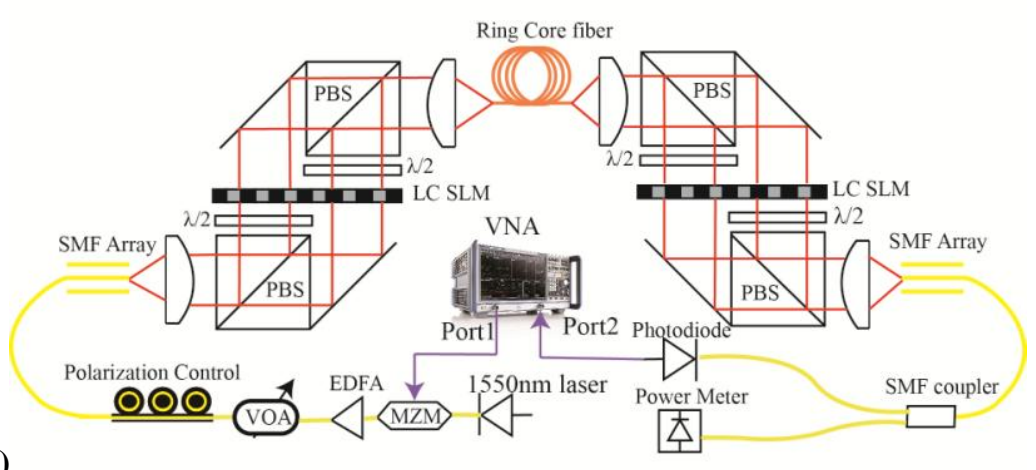

(b)

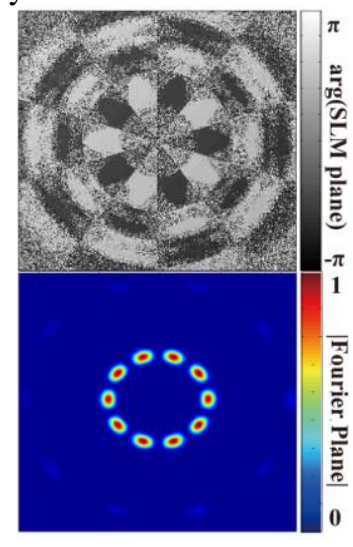

Figure 4 (a) Experimental system used to modally characterize the GI-RCF, (b) an example of phase mask calculated by simulated annealing for LP51a mode of the GI-RCF.
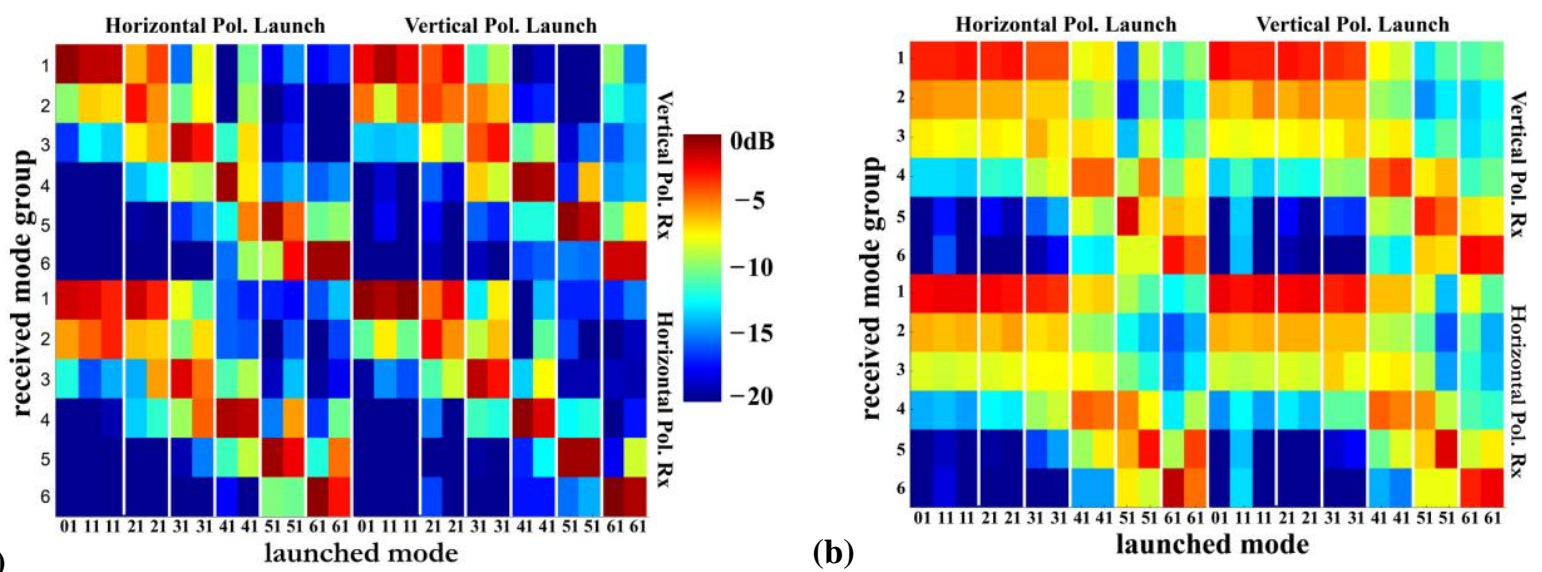

Figure 5 Power distribution of measured mode transfer matrices of (a) 5 meter and (b) 150 meter length of GI-RCF

The use of input and output SMF arrays allows our multiplexer/demultiplexer to support multiple MDM channels simultaneously. However, for modally characterizing the fiber only one channel is used. During the modal 
characterization process, each phase mask in each of the horizontal and vertical polarizations is displayed by the multiplexer SLM one at a time to launch that particular mode. The demultiplexer SLM then decomposes the received modal superposition by displaying each phase mask in each polarization one at a time and measuring the power coupled into the SMF. The relative phase of modes within a polarization is calculated by adding a variable phase shift to each phase mask at the demultiplexer SLM and measuring the power when this is interfered with a reference mode. Following the above process, the mode transfer matrix of the GI-RCF is obtained. Figure 5 shows the power distributions of the measured mode transfer matrices of a 5 meter and 150 meter length of the GI-RCF aggregated by mode groups. As $\mathrm{LP}_{01}, \mathrm{LP}_{11 \mathrm{a}}$ and $\mathrm{LP}_{11 \mathrm{~b}}$ have negligible $\Delta n_{\text {eff }}$, they are considered as one mode group. Observing from the mode matrix, the first three mode groups mix heavily with each other and the last three mode groups exhibit a very good isolation.
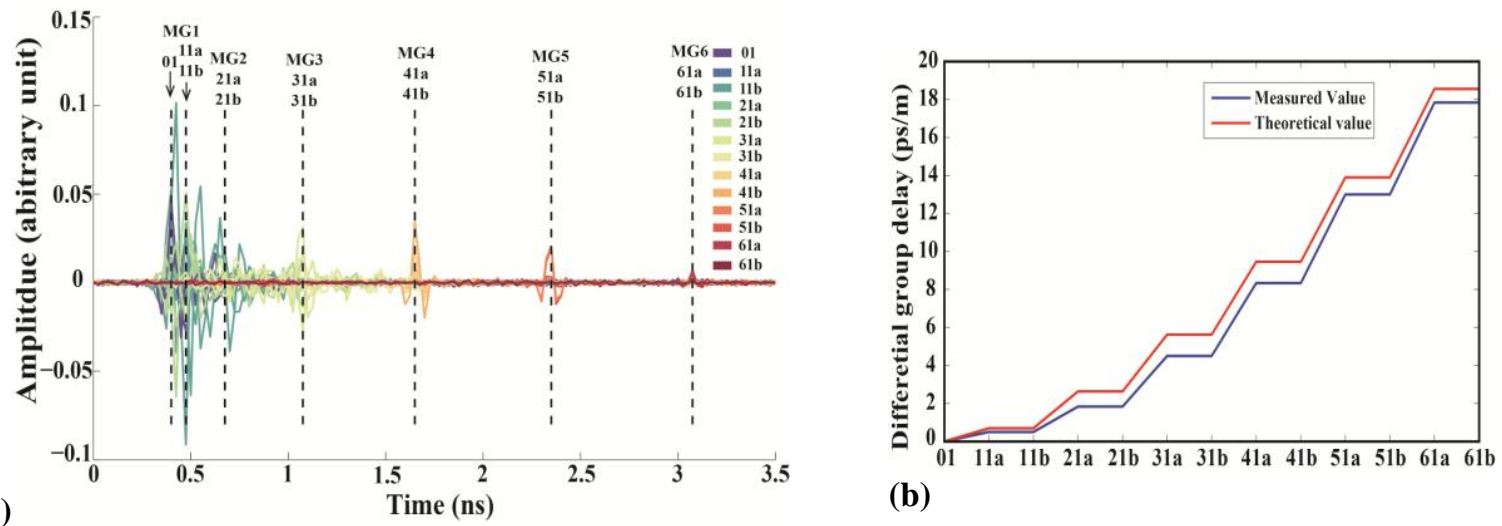

(a)

Time (ns)

(b)

Figure 6 (a) Temporal modal response of the 150 meter length of GI-RCF when each matched pair of launch the detect phase masks are displayed. (b) Comparison of designed and measured differential group delays of the GI-RCF.

The mode transfer matrix measured using the LCoS SLM multiplexer and demultiplexer includes the effect of slight misalignments and uncorrected aberrations, but by measuring time domain data the performance of the actual fiber itself can be analyzed. During the modal characterization process of the $150 \mathrm{~m}$ length of GI-RCF, for each pair of launch and detect mode phase masks (every element in the modal transfer matrix), the VNA takes the frequency response of the GI-RCF. The frequency response of each matched pair of launch and detect mode phase masks (i.e. launching and detecting the same mode) is used to obtain the time response of each mode of the fiber through Fourier transform. The time responses of all the modes of the fiber are superimposed in Figure 6 (a). Distinct modal peaks can be clearly seen and high-order modes produce clean single peaks whereas lower-order modes produce multiple peaks due to heavy mixing, as is expected from the theoretical fiber design. The actual DGD values can be determined from Figure 6 (a) and these closely matched the designed values as shown in Figure 6 (b).

\section{Conclusion}

A graded-index ring-core fiber design supporting 7 LP mode groups with the unique feature, that its first 4 low-order mode-groups are heavily mixed and last 3 high-order mode-groups are highly isolated with $20 \mathrm{~dB}$ isolation, is introduced. This feature of the fiber allows the use of low complexity MIMO processing in MDM systems. We have experimentally characterized the modal properties of the fiber in the spatial and temporal domains using an LCoS SLM based multiplexer and demultiplexer and these were found to be closely matched to our simulation prediction.

\section{References}

[1] Ryf, R.; Randel, S.; Gnauck, A.H.; Bolle, C.; Sierra, A.; Mumtaz, S.; Esmaeelpour, M.; Burrows, E.C.; Essiambre, R.; Winzer, P.J.; Peckham, D.W.; McCurdy, A.H.; Lingle, R., "Mode-Division Multiplexing Over 96 km of Few-Mode Fiber Using Coherent 6 X 6 MIMO

Processing," Lightwave Technology, Journal of , vol.30, no.4, pp.521,531, Feb.15, 2012.

[2] Carpenter, J.; Thomsen, B.C.; Wilkinson, T.D., "Degenerate Mode-Group Division Multiplexing," Lightwave Technology, Journal of , vol.30, no.24, pp.3946,3952, Dec.15, 2012.

[3] Sillard, Pierre; Bigot-Astruc, Marianne; Boivin, D.; Maerten, H.; Provost, L., "Few-mode fiber for uncoupled mode-division multiplexing transmissions," Optical Communication (ECOC), 2011 37th European Conference and Exhibition on , vol., no., pp.1,3, 18-22 Sept. 2011. [4]X.Q. Jin, A. Gomez, D.C. O'Brien, and F.P. Payne, "Influence of Refractive Index Profile of Ring-Core Fibres for Space Division Multiplexing Systems”, IEEE Summer Topicals, (USA, 2014), pp. 178-179 